Staatsräson kontra Utopie? 


\author{
Peter Nitschke
}

\title{
STAATSRÄSON KONTRA UTOPIE?
}

\author{
Von Thomas Müntzer \\ bis zu Friedrich II. von Preußen
}

Verlag J.B. Metzler

Stuttgart - Weimar 
Die Deutsche Bibliothek - CIP-Einheitsaufnahme

Nitschke, Peter:

Staatsräson kontra Utopie? : Von Thomas Müntzer bis zu

Friedrich II. von Preussen / Peter Nitschke. - Stuttgart ; Weimar : Metzler, 1995 ISBN 978-3-476-01344-6

\author{
ISBN 978-3-476-01344-6 \\ ISBN 978-3-476-03623-0 (eBook) \\ DOI 10.1007/978-3-476-03623-0
}

Dieses Werk einschließlich aller seiner Teile ist urheberrechtlich geschützt.

Jede Verwertung außerhalb der engen Grenzen des Urheberrechtsgesetzes ist ohne Zustimmung des Verlages unzulässig und strafbar. Das gilt insbesondere für Vervielfältigungen,

Übersetzungen, Mikroverfilmungen und die Einspeicherung und Verarbeitung in elektronischen Systemen.

(C) 1995 Springer-Verlag GmbH Deutschland

Ursprünglich erschienen bei J.B. Metzlersche Verlagsbuchhandlung und Carl Ernst Poeschel Verlag GmbH in Stuttgart 1995 
Für Annette-Beate

und Christine Sophia 


\section{Inhaltsverzeichnis}

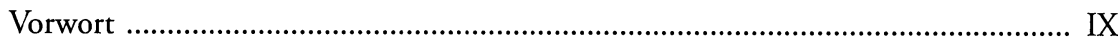

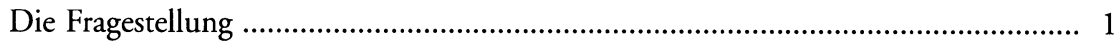

Teil A / Positionsbestimmung:

Forschungsfragen und ihre (bisherigen) Antworten ........................................... 9

I. Vom Umgang mit der Geschichte politischer Ideen ............................... 11

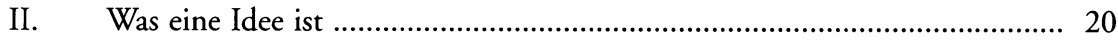

III. Ontologische Dialektik als Methodologie ............................................... 24

IV. Klassifikationsmuster der politischen Phänomene Staatsräson und Utopie .... 34

IV.1 Machiavelli und Machiavellismus ................................................. 35

IV.2 Typologie der Staatsräson ............................................................ 42

IV.3 Phänotypen im historischen Prozeß und ihre Rezeption .................. 48

IV.4 Morus/Platon und die Herrschaft von Nirgendwo ........................ 64

IV.5 Begriff und Impetus von Utopie ...................................................... 73

IV.6 Potentiale des Utopischen in der Prämoderne ............................... 82

IV.7 Staatsräson und Utopie ........................................................... 94

Teil B / Perspektiven:

Deutsche Denker und ihre Bestimmung des Politischen

im Spannungsfeld von Staatsräson und Utopie

I. Thomas Müntzer - oder: das Repräsentationsproblem von wahrer christlicher Herrschaft

II. Johann Oldendorp - oder: die Ordnungsräson der Exekutive ................... 124

III. Melchior von Osse - oder: die Fundierung einer praktischen

Politica Christiana

IV. Johannes Althusius - oder: der Begriff des Politischen als theokratischer Reflex ................................................................................................. 153

V. Johann Valentin Andreae - oder: die Utopie einer antimachiavellistischen Gemeinschaft .................................................................................... 178

VI. Dietrich Reinkingk - oder: das Plädoyer für eine christliche Staatsräson .. 196 
VII. Gottfried Wilhelm Leibniz - oder: die Koinzidenz von Staatsräson und Utopie in der politischen Metaphysik

VIII. Friedrich II. von Preußen - oder: Staatsräson als persönliche Selbstlegitimation

Teil C / Ausblick:

Säkularität und Metaphysik des Politischen

I. Staatsräson als Ideologie ....................................................................... 267

II. Utopie als Optimierung von Wirklichkeit .......................................... 271

III. Die Ideologisierung und Nihilierung der Utopie ..................................... 275

IV. Ende und Anfang des Politischen im Verfall von staatlicher Ordnung ..... 285

Abkürzungsverzeichnis 297

I. Verzeichnis der gedruckten Quellen 298

II. Literaturverzeichnis 301 


\section{Vorwort}

"Unser Übereinkommen aber betraf einen einzigen Hauptpunkt: wie man ein guter Mensch werden kann, der die einem Menschen zukommende Tugend der Seele besitzt, sei es infolge irgendeiner Beschäftigung oder einer Veranlagung oder einer bestimmten Erwerbsart oder eines Strebens oder einer Meinung oder irgendwelcher einmal erworbenen Kenntnisse und ungeachtet dessen, ob einer unserer Bürger männlichen oder weiblichen $G e-$ schlechts ist, ob jung oder alt - daß also auf eben dieses genannte Ziel das ganze Streben das ganze Leben hindurch gerichtet sein muß; und daß niemand, wer er auch sei, erkennen lassen darf, daß er irgend etwas von all dem andern, was dem im Wege steht, höher schätzt, nicht einmal am Ende gar den Staat, wenn es sich als notwendig erweisen sollte, daß der Staat besser zugrunde geht, ehe er das Sklavenjoch auf sich nimmt und sich von schlechteren Leuten lenken läßs, oder daß man den Staat durch freiwillige Verbannung verläßt; denn alles Derartige muß man eher über sich ergehen lassen, als daß man sich eine Verfassung einhandelt, in deren Wesen es liegt, die Menschen schlechter zu machen."

(Platon: Nomoi, 770 c 7 - e 5)

Die übliche Danksagung einmal anders herum: die familiäre Szene nicht an das Ende, sondern an den Anfang. Meiner Frau und meiner Tochter, die mich in der Zeit der Entstehung dieser Arbeit begleitet haben, ist das folgende Werk gewidmet. Dies vor allem auch vor dem Hintergrund des wichtigen Fingerzeigs Dietrich Reinkingks: der status familiae semper est ante rationem statuum de re publica.

Dank bin ich selbstverständlich auch einer Vielzahl von Kollegen schuldig, die mir bei den Reflexionen zu dieser Arbeit, ihren hermeneutischen und inhaltlichen Fragestellungen und Problemen jeweils im Kontext ihres unterschiedlichen Forschungsblickes konkrete oder indirekte Hinweise geben konnten.

An erster Stelle sei hier Prof. Karl Hahn genannt, der sich nicht nur als funktionaler Betreuer einer Habilitationsschrift verstanden hat, sondern im Sinne der klassischen Philosophie als wahrer Anwalt in spiritualibus stets zur Seite war, wenn es etwas Spannendes zu diskutieren galt. Das gilt für den historischen Part in gleicher Weise für Prof. Eco Haitsma Mulier (Amsterdam), bei dem ich einen einjährigen Forschungsaufenthalt verbringen konnte und der mir die spezifischen Eigenheiten des niederländischen Republikanismus in der Prämoderne wie in der Gegenwart verdeutlichte. Nicht zuletzt das Althusius-Kapitel hat hiervon profitiert.

Den beiden Freunden vom Historischen Seminar, Johannes Arndt und Ronald G. Asch, gilt ebenso mein Dank - sowohl für die nächtelangen Diskussionsrunden in Sa- 
chen der prämodernen politischen Theorie wie überhaupt für die Problemerörterungen bei unseren sich phasenweise überlappenden jeweiligen Habilitationsverfahren.

Frau Prof. Nicolette Mout (Leiden) sei ebenso hier erwähnt, weil es nicht zuletzt ihr Wolfenbütteler Sommerseminar im Jahr 1989 gewesen ist, welches im Nachhinein die Initialzündung für die Fragestellung dieser Arbeit lieferte.

Dank gebührt auch den folgenden Professoren, die jeweils ein bestimmtes Kapitel dieser Arbeit zur Kritik gelesen und kommentiert haben: Hans-Jürgen Goertz (Hamburg) für das Müntzer-Kapitel, Michael Stolleis (Frankfurt a.M.) für Oldendorp, Hans Maier (München) für Osse, und Horst Dreitzel (Bielefeld) für Reinkingk. Gerhard Biller M.A. von der Leibniz-Forschungsstelle in Münster hat sich in höchst liebenswerter Weise den Leibniz-Part angeschaut. Ähnliches gilt auch für Prof. Dieter Wyduckel (nunmehr Dresden), dessen Zurverfügungstellung einer bis dato nicht veröffentlichten deutschen Hilfsübersetzung zu Althusius' monumentalem Werk eine große Orientierungshilfe bedeutete.

Anregende Gespräche über Einzelaspekte führte ich mit den Professoren Heinz Duchhardt (nunmehr Mainz), Martin Brecht (Münster), Volker Gerhardt (Berlin) und Robert Bireley (Chicago). Ihnen allen sei diesbezüglich gedankt.

Es sollten an dieser Stelle aber auch die Studentinnen und Studenten meiner Seminare zwischen 1990 und 1993 erwähnt werden: a) weil es nicht unbedingt selbstverständlich ist, sich heutzutage auf solche scheinbar merkwürdigen politischen Diskurse von Staatsräson und Utopie in der Prämoderne einzulassen und b) weil ihre Fragen, ihre Antworten und auch z.T. ihr jeweiliges Unverständnis hermeneutisch wichtig waren für das Zustandekommen dieser Arbeit.

Zu guter Letzt sei hier der Fritz Thyssen Stiftung gedankt, die drei Jahre lang meine Forschungen im Rahmen eines Habilitationsstipendiums großzügig finanziert hat. Ohne diese Hilfe, die auch die überaus ergiebigen Recherchen in den niederländischen Bibliotheken sowie der Herzog-August-Bibliothek in Wolfenbüttel ermöglichte, wäre diese Arbeit nicht zustande gekommen. Sie ist in der vorliegenden Fassung in erster Linie Ergebnis einer methodologischen Vorgehensweise, die in der politikwissenschaftlichen Betrachtung zur Politischen Theorie bzw. Ideengeschichte bis dato nicht vorkommt und insofern Neuland betritt.

Abschließend sei daher noch auf ein Aspekt besonders hingewiesen, nicht zuletzt deshalb, um Mißverständnise von vornherein zu vermeiden. Im folgenden ist oft von der Prämoderne die Rede - und nicht, wie es die Historiker bezeichnen, von der Frühen Neuzeit. Dies hat seinen triftigen Grund: obwohl auch der Terminus Prämoderne den gleichen Zeitraum meint wie der Begriff der Frühen Neuzeit, setzt er ein anderes geschichtsphilosophisches Gedankenbild voraus. Worauf es mir hierbei ankommt, ist die Feststellung, daß wir uns mitnichten in einer Art teleologischer Entwicklung bis zu unserer eigenen politischen Existenz befinden. Die Geschichte, sollte sie etwas beweisen, vermittelt mehr diskontinuierliche Außerachtlassungen im Sinne des Kuhnschen Wissenschaftsparadigmas als sukzessive aufeinander aufbauende Transformationen von Seinsweisen. Insofern erscheint es mir sinnvoll, für die vorliegenden Diskurse von einem prämodernen Verhältnis zu sprechen. Sie sind dies um so mehr, wie auch wir uns nicht mehr in der Moderne befinden. Man mag gegen die postmoderne Philosophie vieles einwenden, deutlich ist mit ihrer Kritik an der klassischen Moderne jedoch geworden, daß strukturell so manches nicht mehr beim Alten ist wie es in den 
theoretischen Grundpositionen der Moderne vertreten wird. Die globalen Erschütterungen in der internationalen Politik, die Erosion von Staatlichkeit in ihrer nationalen Disposition wie auch die Verfallserscheinungen von Gesellschaft dokumentieren einen substantiellen Umwandlungsprozeß, der geradezu nach einer neuen hermeneutischen Position für die Deutung der Dinge verlangt.

Ausgehend von der Einschätzung, daß wir uns mittlerweile in einem postmodernen Universum befinden, hat es elementaren Sinn auf eine Welt zu schauen, die jenseits und vor der Moderne bestand. So können die Fragen und Antworten der politischen Diskurse der Prämoderne z.T. sogar substantielle Hinweise dafür sein, auf was wir uns trotz allem - hier und jetzt - einlassen dürfen. Vielleicht werden wir dabei feststellen, daß die Antworten der Moderne - insbesondere ihres Rollenverständnisses von der Aufklärung - zwar die notwendigen Antworten auf die Probleme der Prämoderne waren, doch dies nur in einem kontingent-historischen Sinne, nicht logischerweise für alle Ewigkeit. 